\title{
Novos registros de Ferrugens (Uredinales) sobre Fabaceae para o Brasil ${ }^{1}$
}

\author{
Isadora Fernandes de França² e Helen Maria Pontes Sotão ${ }^{3,4}$
}

Recebido em 4/04/2008. Aceito em 26/01/2009

RESUMO - (Novos registros de Ferrugens (Uredinales) sobre Fabaceae para o Brasil). Em levantamento realizado na Reserva Biológica do Lago Piratuba, no Estado do Amapá, foram identificadas duas espécies de Uredinales que constituem novos registros para o Brasil: Atelocauda incrustans Arthur \& Cummins e Chaconia alutacea Juel. O gênero Atelocauda representa a primeira citação para a América do Sul. Palavras-chave: Amapá, Basidiomycota, Fungos, Leguminosae, Urediniomycetes

ABSTRACT - (News records of Rust Fungi (Uredinales) on Fabaceae from Brazil). During an inventory undertaken at the Lake Piratuba Biological Reserve, Amapá state, two Uredinales species, Atelocauda incrustans Arthur \& Cummins and Chaconia alutacea Juel, were found and reported for the first time in Brazil. The genus Atelocauda is recorded for the first time in South America.

Key words: Amapá, Basidiomycota, Fungi, Leguminosae, Urediniomycetes

\section{Introdução}

Os fungos causadores de ferrugem em plantas (Uredinales) são parasitas obrigatórios que apresentam grande diversidade e especificidade em relação aos hospedeiros, incluindo musgos, samambaias, gimnospermas e várias famílias de monocotiledôneas e dicotiledôneas, ocorrendo em aproximadamente 260 famílias (Hennen \& Buriticá 1980).

Hennen et al. (2005) reconhecem para o Brasil cerca de 800 espécies de Uredinales, incluídas em 56 gêneros holomórficos e nove gêneros anamórficos. Sobre hospedeiros da família Leguminosae estão referidos 118 espécies de ferrugem, parasitando 48 gêneros de plantas, das quais somente seis tem ocorrência para o estado do Amapá. Citações de espécies de Uredinales para este Estado são escassas, encontrando-se algumas referências (Hennen \& Sotão 1996; 1997; Hennen et al. 1998; Sotão et al. 2001).

O presente trabalho tem por objetivo divulgar a primeira ocorrência de duas espécies de ferrugens no Brasil, procedentes de áreas da Reserva Biológica (REBIO) do Lago Piratuba, no Estado do Amapá. Apresenta descrições morfológicas, material examinado, comentários e ilustrações, ampliando desta forma a distribuição geográfica e o conhecimento sobre a diversidade de espécies de Uredinales na Amazônia.

\section{Material e métodos}

As coletas de amostras de folhas com sintomas de ferrugem (manchas, pústulas, galhas e outras anomalias) foram realizadas em áreas da REBIO do Lago Piratuba, no nordeste do Estado do Amapá $\left(01^{\circ} 10^{\prime}-01^{\circ} 49^{\prime} \mathrm{N}\right.$ e $\left.49^{\circ} 54^{\prime}-50^{\circ} 34^{\prime} \mathrm{W}\right)$, no período de junho e novembro de 2006. As técnicas de coleta e preservação adotadas seguiram Cummins \& Hiratsuka (2003) e Fidalgo \& Bononi (1989). As folhas coletadas foram acondicionadas em sacos plásticos, para posterior prensagem e secagem $\left(50-60{ }^{\circ} \mathrm{C}\right)$ em estufa a gás.

A identificação dos espécimes foi feita com base nos hospedeiros e nas análises das microestruturas dos fungos. Para observação microscópica do material foram montadas lâminas semipermanentes de soros e esporos em solução de lactofenol, posteriormente aquecidas. Para o exame da superfície das estruturas fúngicas em microscopia eletrônica de varredura (MEV), foram cortados fragmentos das folhas contendo soros. Estas amostras, e também esporos, foram depositados em suportes de alumínio com $10 \mathrm{~mm}$ de diâmetro utilizando-se fita adesiva de carbono e metalizados com ouro, utilizando-se corrente de $25 \mathrm{~mA}$, em atmosfera de ar de 1.10-2 mbar durante 02'30'”. As imagens foram obtidas em MEV marca LEO, modelo 1450VP, com aceleração de voltagem de $10 \mathrm{kV}$, e registradas em modo digital, em formato "tiff". Literatura especializada com descrições de espécies e chaves de identificação foi utilizada: Cummins (1978), Cummins \& Hiratsuka (2003), Hennen et al. (2005), Ono (1984) e Walker (2001). As descrições taxonômicas das espécies foram feitas a partir das características dos espécimes estudados e complementadas com descrições de literatura, conforme apresentado nos comentários, e as ilustrações das microestruturas foram feitas em fotomicroscópio (MO) e em microscopia eletrônica de varredura (MEV).

As amostras estão depositadas nos Herbários João Murça Pires (MG), do Museu Paraense Emílio Goeldi, Belém (PA) e Herbário Amapaense (HAMAB), do Instituto de Estudos e Pesquisas do Estado do Amapá, Macapá (AP).

\section{Resultados e discussão}

Nas amostras coletadas foram identificadas duas novas ocorrências de Uredinales para o Brasil, pertencentes às famílias Chaconiaceae (Chaconia alutacea Juel) e Pileolariaceae (Atelocauda incrustans Arthur \& Cummins), sendo que o gênero Atelocauda representa primeiro registro para a América do Sul.

Atelocauda incrustans Arthur \& Cummins, Annales Mycologici 31, 41. 1933.

Fig. 1-2, 4-8

$\equiv$ Pileolaria incrustans (Arthur \& Cummins) Thirum. \& F. Kern, Bulletin of the Torrey Botanical Club 82, 105. 1955.

Lesões anfígenas, geralmente na nervura central ou ao lado das veias principais e neste caso ao longo destas, $2-4 \mathrm{~mm}$ compr. Télios agrupados em uma única pústula maior ou em grupos menores dispersos, anfígenos, irrompentes, com a epiderme circundando o soro; teliósporos (20-)22-30x (15-)18-22 $\mu \mathrm{m}$, geralmente elipsóides a obovóides, parede

\footnotetext{
Parte da Dissertação de Mestrado da primeira Autora

2 Universidade Federal Rural de Pernambuco, DEPA, Programa de Pós-Graduação em Fitopatologia, Dois Irmãos, Recife, PE, Brasil

3 Museu Paraense Emílio Goeldi, MPEG, Coordenação de Botânica, Belém, PA, Brasil

4 Autor para correspondência: helen@museu-goeldi.br
} 
uniforme 1-2 $\mu \mathrm{m}$ espes., com apêndices cúbicos ou divididos apicalmente, sendo maiores e mais numerosos no ápice do esporo e geralmente arranjados em linhas na porção basal, marrom-canela escuro a marrom-dourado, com pedicelos quebradiços na maioria dos esporos.

Material estudado: BRASIL. Amapá: Amapá, REBIO do Lago Piratuba, Fazenda Igarapé Santa Cruz, 01²0’33’'N 50¹4'47'W, sobre Derris glabrescens (Benth.) J.F. Macbr. (Fabaceae), 4/VII/2006, França, I. I-29 (MG, HAMAB); 6/VII/2006, França, I. I-32 (MG, HAMAB).

Distribuição geográfica: segundo Walker (2001) esta espécie era conhecida apenas no Panamá, sendo este o primeiro registro para o Brasil.

Comentários: nas amostras analisadas foram observados apenas télios e teliósporos. A descrição dos demais estágios (O, I, II) é apresentada em Walker (2001).

Segundo Walker (2001), o gênero Atelocauda tem como tipo a espécie Atelocauda incrustans, a qual foi descrita a partir de dois espécimes coletados no Panamá, em 1920, sobre folhas de Lonchocarpus sp. (Fabaceae). O espermogônio subcuticular, sem paráfises (tipo 7 de Hiratsuka \& Hiratsuka,1980) e os télios subepidermais que produzem teliósporos unicelulares, marrons, pedicelados e com ornamentações cubiformes caracterizam o gênero.

Thirumalachar \& Mundkur (1949) aceitaram o gênero Atelocauda como válido. Posteriormente, Thirumalachar \& Kern (1955) colocaram A. incrustans como sinônimo de Pileolaria, denominando-a P. incrustans (Arthur \& Cummins) Thirum. \& F. Kern, proposta aceita por Cummins (1978).

O gênero permaneceu monoespecífico até que Cummins \& Hiratsuka (1983) o revalidaram como Atelocauda e transferiram para este gênero três espécies de Uromyces que ocorrem sobre plantas do gênero Acacia: U. bicinctus
McAlpine, $U$. digitatus G. Winter e $U$. koae Arthur), recombinados respectivamentes para $A$. bicincta (McAlpine) Cummins \& Y. Hirats., A. digitata (G. Winter) Cummins e Y. Hirats. A. koae (Arthur) Cummins e Y. Hirats. Em seguida, Ono (1984) recombinou uma outra espécie de Uromyces que também ocorre sobre Acacia (Uromyces hyalosporus Sawada), como A. hyalospora (Sawada) Ono. Gardner (1991) descreveu ainda uma nova espécie deste sobre Acacia, A. angustiphylloda D.E. Gardner.

A partir de revisão do gênero Atelocauda com seis espécies conhecidas, Walker (2001) redefiniu este gênero, descrevendo o uredínio aecidióide de A. incrustans e a nova espécie A. shivasii J. Walker sobre Ormosia (Fabaceae), da Austrália. Neste trabalho, o autor transferiu as cinco espécies de Atelocauda sobre Acacia para um novo gênero, Racospermyces J. Walker. Mais recentemente Scholler M. \& Aime (2006), transferiram estas cinco espécies de Racospermyces para o gênero Endoraecium.

A planta hospedeira dos espécimes estudados foi identificada como Derris glabrescens, que possui como basiônimo Lonchocarpus glabrescens Benth., o mesmo gênero de hospedeiro dos espécimes de A. incrustans do Panamá.

Este é o primeiro registro do gênero Atelocauda para a América do Sul.

Chaconia alutacea Juel, Bih. Till K. Svenska Vet.-Akad. Handl. 23: 12. 1897.

Fig. 3, 9

=Uromyces albescens Syd. \& P. Syd., Ann. Mycol. 14: 66. 1916.

= Maravalia albescens (Syd. \& P. Syd.) Dietel, Ann. Mycol. 22: 270.1924.

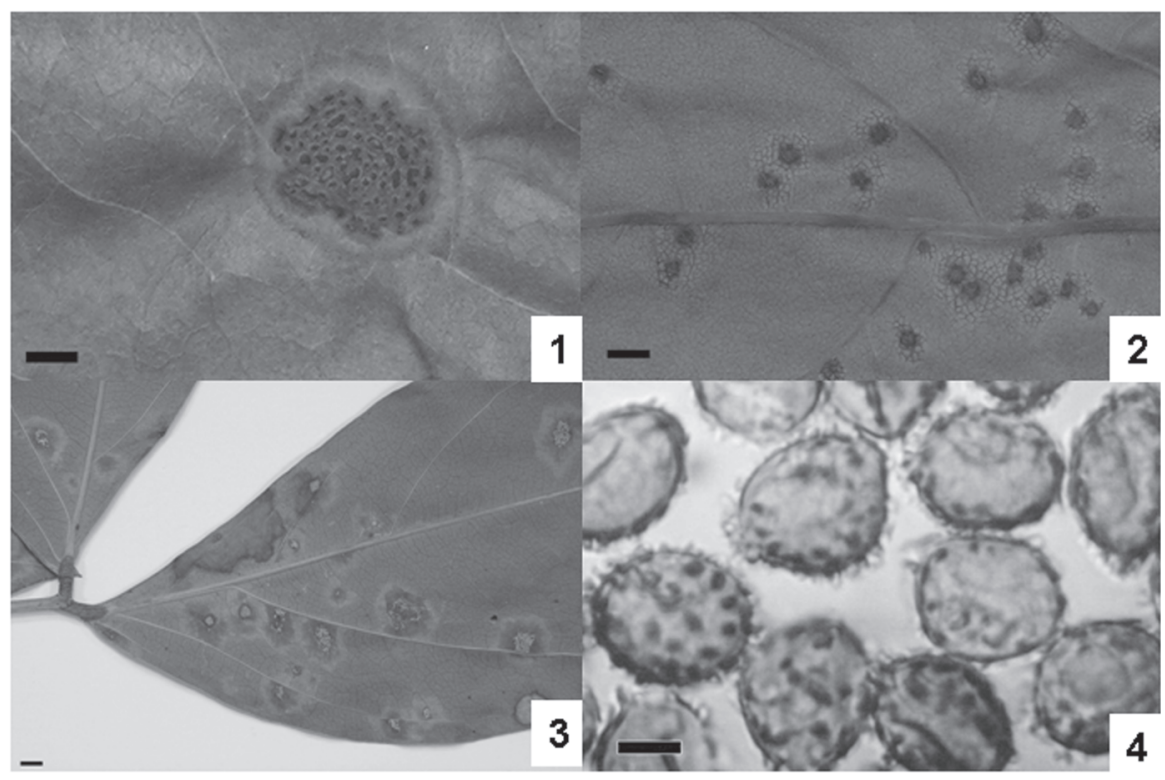

Figuras 1-2. Folíolos de Derris glabrescens (Benth.) J.F. Macbr., parasitados por Atelocauda incrustans Arthur \& Cummins: 1. Télios agrupados em uma única pústula maior. 2. Télios organizados em grupos menores, dispersos. Barras $=1 \mathrm{~mm}$. 3. Detalhe de folhas de Pithecellobium inaequale (Humb. \& Bonpl. ex Willd.) Benth., com os sintomas causados por Chaconia alutacea Juel. Barra $=2 \mathrm{~mm}$. 4. Atelocauda incrustans Arthur \& Cummins. Teliósporos vistos ao microscópio de luz em foco médio. Barra $=$ ca. $8,7 \mu \mathrm{m}$. 


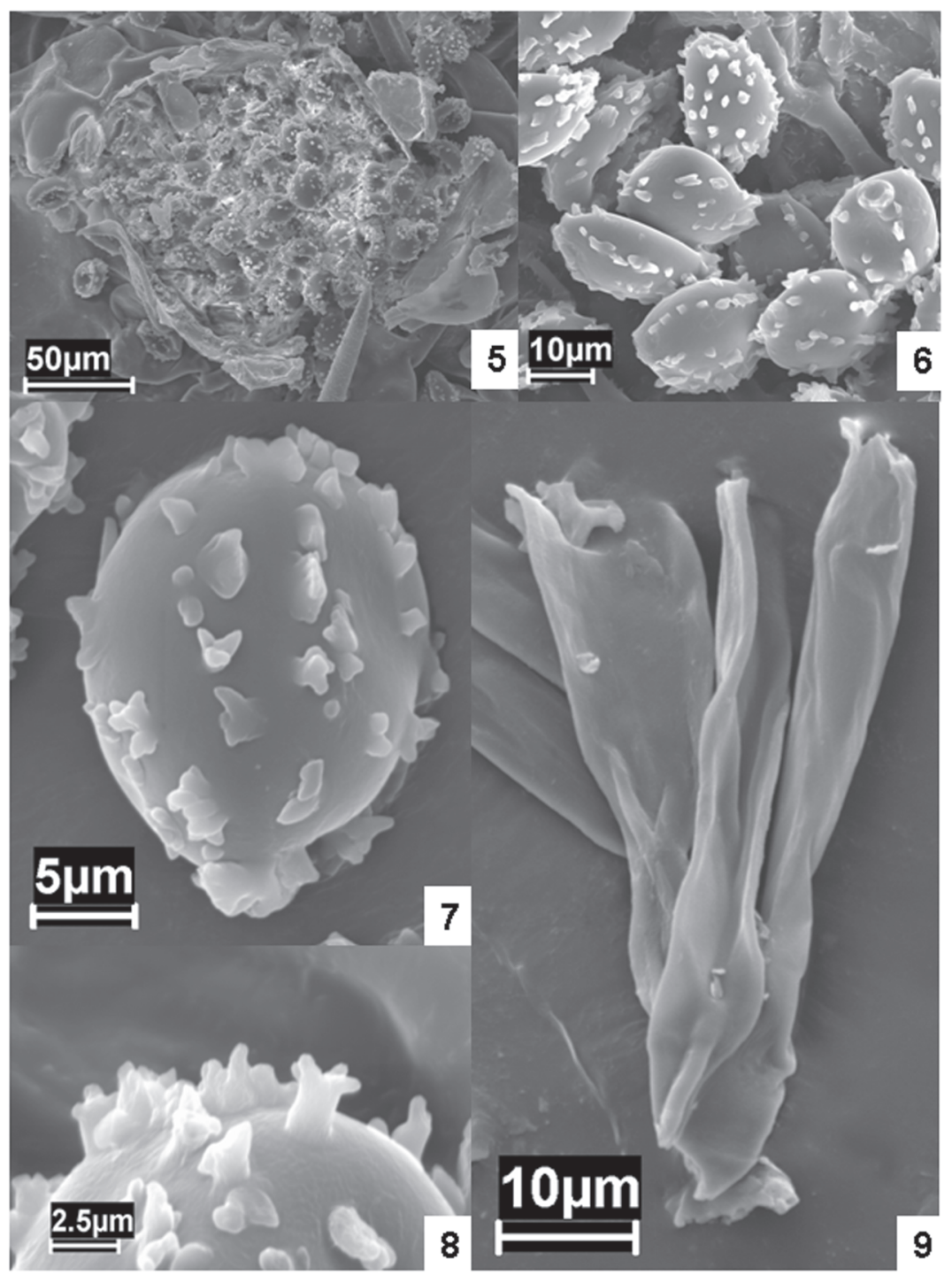

Figuras 5-8. Atelocauda incrustans Arthur \& Cummins: 5. Télio visto ao microscópio eletrônico de varredura (MEV). Barra = ca. 50 $\mu \mathrm{m}$. 6-7. Teliósporos ao MEV mostrando a ornamentação da parede. Barras $=$ ca. $10 \mu \mathrm{m}$ (7), $5 \mu \mathrm{m}$ (8). 8. Detalhe de um teliósporo mostrando os apêndices que geralmente são cúbicos ou divididos (ramificados) apicalmente. Barra $=$ ca. 2,5 $\mu \mathrm{m}$. Figura 9. Chaconia alutacea Juel. Teliósporos ao MEV. Barra $=$ ca. $10 \mu \mathrm{m}$.

Lesões anfígenas, causando manchas foliares, de 2 a $5 \mathrm{~mm}$. Télios hipófilos, , isolados ou em grupos pequenos, subepidermais, irrompentes, teliósporos (probasídios) clavados a cilíndricos, 40-70×10-18 $\mu \mathrm{m}$, parede fina, hialina; metabasídios formados por elongações apicais contínuas do probasídio.

Material estudado: BRASIL. Amapá: Amapá, Fazenda Macarry, 01 ${ }^{\circ} 47^{\prime} 32^{\prime \prime}$ N-50 33'04'W, sobre Pithecellobium inaequale (Humb. \& Bonpl. ex Willd.) Benth. (Fabaceae), 12/XI/2006, Lobato, L.C. \& Sanjuan, P. LC38 (MG, HAMAB).

Distribuição geográfica: esta espécie tem distribuição conhecida para Belize, Paraguai e Peru (Ono \& Hennen 1983).

Comentários: Chaconia alutacea é a espécie tipo do gênero Chaconia. Segundo Hennen et al. (2005) os espermogônios e écios são conhecidos apenas para o
Belize, e não foram encontrados no material examinado. Apresenta-se a complementação da descrição com base nas informações de Cummins (1978) e Ono (1984), "Espermogônios anfígenos, subcuticulares, lenticulares a hemisféricos. Écios hipófilos, agrupados em algumas lesões hipertrofiadas opostas aos espermogônios, subepidermais em origem, irrompentes, eciósporos pedicelados, obovóides a elipsóides, 20-26×16-20 $\mu \mathrm{m}$, parede ca. $1 \mu \mathrm{m}$ de espessura, marrom-canela, equinulada. Uredínios desconhecidos".

Silveira (1951) cita Uromyces albescens como conhecida para o Brasil, porém não referencia a procedência do material. Hennen et al. (2005) ressaltam que este registro precisa ser confirmado, embora seja esperado, devido à proximidade dos locais conhecidos, no Peru e Paraguai. Dessa forma, este se trata do primeiro registro da espécie para o Brasil, apresentando as informações pertinentes. 
Hennen et al. (2005) citam oito espécies sobre o gênero Pithecellobium: Chaconia alutacea Juel, Diorchidium acanthostephum Syd. \& P. Syd., Ravenelia minuta Syd., Ravenelia pileolarioides Syd., Ravenelia pithecolobii Arthur, Uredo amazonica (Syd.) J.W. Baxter, Uredo bomfinensis Henn., Uredo pithecolobii Henn. Chaconia alutacea diferencia-se das demais espécies citadas por apresentar teliósporos unicelulares, com parede lisa, sésseis, lateralmente livres, produzidos em pequenos grupos sobre células esporogênicas.

Ono (1984), em sua monografia sobre o gênero Maravalia, descreve M. pallida Arthur \& Thaxt. ex Arthur, sobre o gênero Pithecellobium, a partir do material tipo de Trinidad. Maravalia pallida pode ser diferenciada de Chaconia alutacea por apresentar pedicelos longos, persistentes, com até $40 \mu \mathrm{m}$ de comprimento.

Com este estudo mais duas espécies são adicionadas à lista de ferrugens do Brasil e o gênero Atelocauda é registrado pela primeira vez para América do Sul. Esta contribuição confirma a importância de inventários em áreas inexploradas ou de megabiodiversidade como a Amazônia, possibilitando a descoberta de novos registros e táxons para a região tropical.

\section{Agradecimentos}

Os autores agradecem ao Programa ARPA/MMA/IBAMA pelo financiamento, coordenação e apoio logístico; Ao IEPA e MPEG pelas facilidades e infraestrutura oferecidas; ao Dr. Joe F. Hennen pela bibliografia cedida; aos colegas Salustiano Villar, Luís Carlos Lobato, Carlos Alberto Silva e Priscila Sanjuan, pelo apoio nas coletas e identificações Botânicas; ao CNPq pela concessão de bolsa de mestrado ao primeiro autor.

\section{Referências bibliográficas}

Cummins, G.B. 1978. Rust Fungi on Legumes and Composites in North America. Tucson, University of Arizona Press.

Cummins, G.B. \& Hiratsuka, Y. 1983. Illustrated Genera of Rust Fungi. Revised Edition. St. Paul, The American Phytopathological Society.
Cummins, G.B.; Hiratsuka, Y. 2003. Illustrated Genera of Rust Fungi. 3 ed. St. Paul, The American Phytopathological Society.

Fidalgo, O. \& Bononi, V.L.R. 1989. Técnicas de coleta, preservação e herborização de material botânico. São Paulo, Instituto de Botânica.

Gardner, D.E. 1991. Atelocauda angustiphyllodia n. sp., a microcyclic rust on Acacia koa in Hawaii. Mycologia 83: 650-653.

Hennen, J.F. \& Buriticá, P. 1980. A brief summary of modern rust taxonomic and evolutionary theory. Reports of the Tottori Mycological Institute 18: 243-256.

Hennen, J.F.; Figueiredo, M.B.; Carvalho Jr, A.A. \& Hennen, P.G. 2005. Catalogue of plant rust fungi (Uredinales) of Brazil. Disponível em: <http://www.jbrj.gov.br> (Acesso em: 15/08/2005).

Hennen, J.F. \& Sotao, H.M.P. 1996. New species of Uredinales on Bignoniaceae from Brazil. SIDA 17: 173-184.

Hennen, J.F. \& Sotao, H.M.P. 1997. Aecidium Maprouneae Var. noncrassatum sobre Maprounea sp. novo táxon do Estado do Amapá, Brasil. Fitopatologia Brasileira 22: 444-447.

Hennen, J.F.; Sotao, H.M.P. \& Hennen, M. 1998. The Genus Diorchidium in Neotropics. Mycologia 90: 1079-1086

Hiratsuka, Y. \& Hiratsuka, N. 1980. Morphology of spermogonia and taxonomy of rust fungi. Reports of the Tottori Mycological Institute 18: 257-268

Ono, Y. 1984. A Monografia of Maravalia (Uredinales). Mycologia 76: 892-911

Ono, Y. \& Hennen, J.F. 1983. Taxonomy of the Chaconiaceous genera (Uredinales). Transactions of the Mycological Society of Japan 24: 369-402.

Silveira, V.D. 1951. Elementos de Fitopatologia. Agronomia 10: $1-44$

Scholler, M. \& Aime M.C. 2006. On some rust fungi (Uredinales) collected in an Acacia koa - Metrosideros polymorpha woodland, Mauna Loa Road, Big Island, Hawaii. Mycoscience 47: 159-165.

Sotão, H.M.P.; Hennen, J.F. \& Cavalcanti, M.A. 2001. Uredinales do Estado do Amapá: I - Gênero Puccinia. Boletim do Museu Paraense Emílio Goeldi - Série Botânica 17: 107-159.

Thirumalachar, M.J. \& Kern, F.D. 1955. The rust genera Allotelium, Atelocauda, Coinostelium and Monosporidium. Bulletin of the Torrey Botanical Club 82: 102-107.

Thirumalachar, M.J. \& Mundkur, B.B. 1949. Genera of rusts I. Indian Phytopathology 2: 65-101.

Walker, J. 2001. A revision of the genus Atelocauda (Uredinales) and description of Rancospermyces gen. nov. for some rusts on Acacia. Australasian Mycologist 20: 3-28. 